The Pharmacogenomics Journal (2006) 6, 2. doi:10.1038/sj.tpj.6500368

\section{Barrriers to testing}

Pharmacogenetic tests are not commonly used owing to a variety of practical roadblocks. Woelderink and colleagues (pp 3-7) present their results after surveying respondents' usage of two pharmacogenetic tests in four European countries. The first, which tests human epidermal growth factor receptor 2 (HER2) overexpression, predicts the usefulness of trastuzumab treatment of breast cancer and was backed by an industry-sponsored educational campaign. The second, which tests for thiopurine methyltransferase (TPMT) activity, identifies poor metabolizers of immunosuppressants and had a small, mostly unsubsidized market. Eighty-four percent of respondents consistently tested for HER2 overexpression, while only $12 \%$ tested for TPMT activity. Reported implementation obstacles included cost, perception, social and legal reasons.

\section{Alzheimer target}

Alzheimer's disease (AD) affects the brain and behavior and an estimated 4.5 million Americans suffer from this debilitating disorder. Fumagalli and colleagues (pp 8-15) present a comparison of drugs used to clinically manage neurodegenerative disease. Of the many types of pharmacotherapeutics considered, most seem to interfere with brain-derived neurotrophic factor (BDNF) expression in the forebrain (the area affected by $A D$ ). The authors conclude that modulation of BDNF levels will help foster drug discovery, especially for therapeutic intervention of areas affected but not completely damaged. Further reasoning that therapy could some day be directed at specific areas of the brain affected by AD.

\section{Personalized treatment}

Genetic makeup can affect the metabolism, mechanism and action of therapeutic compounds especially in the treatment of serious disease such as cancer and hypertension. In this review, Shastry (pp 16-21) summarizes recent studies that compare the relationship of genetic variants and drug responses. The author discusses how the study of one gene and the study of many genes in combination with geographic and ethnic factors further the concept of individualized medicine, thus preventing adverse drug reactions (or no action). Although the field is still considered new, Shastry maintains that personalized treatment can only become a clinical reality with further study and public acceptance.

\section{High blood pressure}

The treatment goal for hypertension is to lower blood pressure to levels that will decrease the risk of cardiovascular-related complications. In a 10-year study of 625 patients, Schelleman et al. (pp 22-26) evaluated the relationship of polymorphisms in those managed with diuretics, beta-blockers or ACE inhibitors. The authors investigated 36 possible gene-gene-drug interactions and noted four that were significant in lowering blood pressure levels. Of the four, it was found that patients with the GNB3 TT polymorphism had lower systolic levels while treated with a diuretic. The authors suggest that the effects of SNPs and gene-gene-drug interactions had a small effect in those with GNB3 and further investigations with a larger sample size are needed.

\section{Depression in Taiwan}

Hong and colleagues (pp 27-33) examined a typical selective serotonin reuptake inhibitor, fluoxetine, and the mechanisms of its intended therapeutic effect. Researchers initiated association studies involving several polymorphisms related to fluoxetine response, particularly the C-1019G polymorphism on the HTR1A gene. Two hundred and twenty-four ethnic Chinese adults diagnosed with major depression were treated with fluoxetine for four weeks. Response was best predicted by age and the genotypes of SERTPR and HTR1A; combined, these factors accounted for $13.2 \%$ of the inconsistency of response. The authors recommend further study to include a longer treatment evaluation, a review of more genes and better accounting for other factors like placebo effect and patient histories to give a more complete picture of genetic connections to depression.

\section{Alcohol withdrawal}

For alcohol-dependent adults, withdrawal can sometimes induce epileptic seizures and delirium tremens. Preuss et al. (pp 34-41) investigated the expression of functional polymorphisms of the KA receptor subunit gene GRIK3 coding for GluR7 (the GRIK3 gene is found in a region associated with alcoholism on chromosome 1) in patients whose histories include these withdrawal side effects. Of 233 alcohol-dependent patients, it was found that the GRIK3 polymorphism had no significant effect on developing alcoholism, nor could it be determined to affect seizures. However, $13 \%$ of patients carrying one Ser 310 allele experienced delirium tremens, as did $22 \%$ of those homozygous for the allele. These results indicate no correlation between delirium tremens and seizures, despite their co-occurrence during alcohol withdrawal. However, the GRIK3 polymorphism does appear related to delirium tremens.

\section{SNPs and schizophrenia}

The effectiveness of atypical antipsychotic drugs used to treat schizophrenia may be connected to SNPs affecting dopamine and serotonin G-protein-coupled receptors (GPCRs). Davies and colleagues (pp 42-51) sought to relate drug actions to particular GPCRs, specifically considering functional SNPs that were mapped to the serotonin $5-\mathrm{HT}_{2 \mathrm{~A}}$ receptor. The authors found that these related SNPs can affect drug response up to twofold in vitro, as in the case of the $1197 \mathrm{~V} 5-\mathrm{HT}_{2 \mathrm{~A}}$ receptor's affinity for aripiprazole, and increase antagonist potency 10 -fold, as with clozapine at the receptor. They conclude that specific SNPs had an effect on receptor responses to specific atypical antipsychotic drugs, but none had an effect to all of the drugs.

\section{Dosage based on UGT1A}

Polymorphism UGT1A1*28 is considered a predictor of toxicity in those treated with tranilast, an anti-keloid/anti-allergy agent, or irinotecan, an anticancer drug. In an effort to improve these drugs' efficacy, Maitland et al. (pp 52-62) compared the UGT1A sequence in five species of mammals. After performing a resequencing survey of identified segments, researchers found new coding and noncoding variants. They then examined patterns of common and rare variants within the same gene in 72 people across three population samples and found the frequency of the same allele in similar populations. This study suggests that future genotype-adjusted dosages may help establish safe and effective treatment.

\section{More on UGTS}

Drug metabolism is affected by genetic polymorphisms of UGTs and is the subject of many drug efficacy studies. Saeki et al. (pp 63-75) report on their haplotype organization of the UGT1A gene complex in Japanese subjects. The complex was organized into five blocks and linkage combinations of SNPs were identified. The scientists compared haplotypes to other ethnic groups to confirm results, and found many linkages. As glucuronidation plays a critical part in normal body detoxification, genetic variants that lead to altered expression of UGTs are likely to have some pharmacological consequences in the Japanese. 